\title{
L'HOMME L'Homme
}

Revue française d'anthropologie

157 | janvier-mars 2001

Représentations et temporalités

\section{Christopher Pinney, Camera Indica. The Social Life of Indian Photographs}

London, Reaktion Books, 1997, 246 p., bibl., index, 127 ph.

\section{Emmanuel Grimaud}

\section{OpenEdition}

\section{Journals}

Édition électronique

URL : http://journals.openedition.org//homme/5686

DOI : 10.4000/lhomme.5686

ISSN : 1953-8103

Éditeur

Éditions de l'EHESS

Édition imprimée

Date de publication : 1 janvier 2001

Pagination : 267-270

ISBN : 2-7132-1357-6

ISSN : 0439-4216

Référence électronique

Emmanuel Grimaud, «Christopher Pinney, Camera Indica. The Social Life of Indian Photographs »,

L'Homme [En ligne], 157 | janvier-mars 2001, mis en ligne le 23 mai 2007, consulté le 24 septembre 2020. URL : http://journals.openedition.org//homme/5686 ; DOI : https://doi.org/10.4000//homme. 5686

Ce document a été généré automatiquement le 24 septembre 2020

(c) École des hautes études en sciences sociales 


\title{
Christopher Pinney, Camera Indica. The Social Life of Indian Photographs
}

\author{
London, Reaktion Books, 1997, 246 p., bibl., index, 127 ph.
}

\section{Emmanuel Grimaud}

1 on DoIT à David MacDougall ${ }^{1}$ d'avoir attiré le regard des anthropologues sur le terrain exceptionnel que constituent en Inde, du point de vue d'une sociologie des identités en formation, les pratiques quotidiennes de la photographie. C'est l'« écologie complexe et changeante de la photographie » dans l'Inde coloniale et postcoloniale que Christopher Pinney a choisi d'explorer dans Camera Indica, prolongeant ainsi les travaux classiques de Barthes et de Bourdieu ${ }^{2}$ sur ses usages sociaux. L'auteur rend hommage au premier par le titre, qui fait écho à Camera Lucida, traduction anglaise de La Chambre Claire, et renouvelle l'attention qu'il accorde à l'image dans la construction du sujet. Il emprunte au second le souci de situer son objet dans un champ qui ne cesse de se composer à travers ses usages coloniaux et postcoloniaux. Il revendique enfin son lien à la sociologie des objets d'Arjun Appadurai ${ }^{3}$ en considérant les images comme des acteurs à part entière. À la frontière de l'histoire sociale de l'art, de l'ethnologie et de la sémiotique, la microsociologie des images proposée par Pinney a le mérite de ne pas faire disparaître son objet derrière l'outillage de la sociologie critique ou de la sémiologie, attentive qu'elle est à sa "circulation historique concrète" (Appadurai). Les images commentées par leurs praticiens constituent des objets "imbriqués " (entangled objects) ${ }^{4}$, des espaces où se déploient différentes anthropologies (il y est question du corps et du visage, de l'intériorité et de l'apparence, de l'identité) qu'il présente comme autant d'histoires parallèles : celle de l'identification photographique de ses sujets opérée par l'administration coloniale britannique, celle du développement des studios indigènes de photographie, celle des pratiques contemporaines des habitants d'une ville de l'Inde centrale, Nagda, où la photographie est un support de rituel, de mémoire et de distraction par la médiation de laquelle se reconfigurent les identités.

2 La préoccupation de l'administration coloniale concernant l'identité de ses sujets s'est traduite, dès le début $\mathrm{du} \mathrm{xIX}^{\mathrm{e}}$ siècle, par leur fixation photographique. Les premiers 
clichés d'amateurs anglais des Bengal et Madras Photographic Societies précèdent l'institution d'un "Orient bon à penser pour l'académie, et bon à montrer pour la muséographie» (p.38), avant que la photo soit pleinement exploitée comme instrument d'identification des « races et catégories sociales». Pinney distingue deux « paradigmes » dans cette évolution : le « paradigme d'urgence » (salvage paradigm), qui consiste à capturer les traces des groupes en voie d'extinction, et le «paradigme du détective" (detective paradigm), grâce auquel on peut élaborer des repères visuels d'identification des castes (p.45). Au fur et à mesure de sa consolidation comme instrument de pouvoir servant à répertorier, selon la nomenclature de l'administration coloniale, «tous les stades connus de la culture primitive côtoyant une administration du type le plus moderne » (p. 46), la photographie se préoccupe de moins en moins des individus et de plus en plus des groupes. Le visage et le costume deviennent des marqueurs de la tribu ou de la caste. Du «Birman » à la «Beauté Madrasi » en passant par le «Gujar Typique » ou le «Banyan malhonnête mais utile », s'élaborent peu à peu des grilles de classification où marques corporelles et statuts sociaux renvoient les uns aux autres. À la fin du XIX ${ }^{e}$ siècle prolifèrent les éditeurs de cartes postales représentant les indigènes, les «typical pictures of Indian Natives ».

3 Autrefois objets de connaissance, les catégories professionnelles - du blanchisseur au balayeur - trouvent une fonction au sein du Raj: le fonctionnalisme succède au primitivisme, faisant des sujets indiens de l'Empire des «individus médiatisés par des relations de classe à l'intérieur des structures de pouvoir coloniales » (p. 57). Si l'intérêt pour la diversité des populations locales a perduré tant qu'elles offraient à l'État britannique une « infrastructure à son service» (p. 59), c'est que « les signes extérieurs des corps indiens étaient objectivés en tant que support de l'identité sociale » (p.62). Dans ce contexte, la photographie et son association à l'anthropométrie des groupes ne pouvait être que dépassée comme outil d'authentification (weapon of penetrating certainty) par la technique des empreintes digitales, moyen d'identification plus précis mis au point par l'État colonial sur le terrain indien avant d'être utilisé en métropole.

C'est à un tout autre usage de la photographie que nous convie le chapitre II qui retrace l'histoire des studios « indigènes » entre Indore et Bombay, et dans lesquels, grâce à la photo, «on pouvait accéder à une identité supérieure, plus intense» (p.74). Le contraste, par rapport à la logique précédente, est éloquent : les images des studios ne se préoccupent pas de fixer les identités dans des catégories closes, mais manipulent un répertoire de signes permettant de représenter différentes " possibilités d'être ». Loin d'enfermer les photographies et leurs praticiens dans une dichotomie culturaliste, Pinney souligne que ces studios étaient souvent mixtes, avec des clientèles britanniques, ou se dotèrent de propriétaires indiens sans que cela ait une incidence sur le contenu des photos. S'opposant au cliché mis au service du pouvoir colonial, la photo de studio ne révèle pas l'« existence d'une âme indienne inaltérable » (p. 96), mais constitue une tentative de formulation d'une identité visuelle contemporaine.

5 L'auteur décrit ainsi l'évolution d'un projet singulier mené au début du $\mathrm{xx}^{\mathrm{e}}$ siècle par le théosophe G. S. Arundale à Indore : La Galerie des Grands Hommes (The Picture Gallery of Greatness; p. 102), où les visiteurs étaient invités à contempler (darshan) ${ }^{5}$ les célébrités dont le visage reflétait des qualités morales. Combinant une tradition locale de l'image et la notion victorienne de portrait (p. 105), l'entreprise faisait appel à deux langages contradictoires : alors que le darshan supposait l'invocation d'une image générique dont l'efficacité tenait à la reconnaissance par le dévot de certains traits typologiques, la 
moral physiognomy du portrait victorien reposait sur la visibilité des qualités intérieures d'un personnage singulier. Plus spectaculaire que populaire, le projet d'Arundale, tout en inscrivant de façon unique la photo au sein du rituel, partageait avec la photographie victorienne l'idée que le corps et le visage peuvent refléter des qualités intérieures, voire l'essence d'un individu.

6 C'est encore à une autre " déchiffrabilité » du corps que les pratiques des habitants de Nagda nous invitent dans le troisième et dernier chapitre. L'auteur situe l'usage contemporain du portrait à Nagda au croisement de plusieurs champs. Naviguant de la muséographie coloniale aux studios contemporains, entre l'individu et la famille, la ville et le village, les albums personnels et les vidéos de mariage, la salle de cinéma et le temple domestique, Pinney évite le raccourci culturaliste dont a été victime Judith Mara Gutman ${ }^{6}$, qui consiste à ramener la photo indienne à des invariants empruntant pour l'essentiel à l'iconographie rituelle. La pratique photographique s'éclaire par les décalages et les transferts qu'elle opère constamment au cours de son histoire, du religieux au profane, du cinéma à la photo, menant jusqu'à l'espace créatif et foisonnant des studios d'aujourd'hui.

On voit ainsi comment les studios de Nagda retravaillent les images religieuses dans leurs montages, utilisant pour les albums de mariage les mêmes procédés que pour les icônes. Mais celles-ci ne sont pas les seules sources d'inspiration : un couple se met en scène dans un téléviseur, un autre entre les ailes d'un papillon, entre deux fleurs ou deux étoiles, donnant ainsi forme à leur compatibilité ; le portrait d'un jeune marié se trouve décuplé le long d'un négatif de film, exemple parmi d'autres de la créativité qui anime les studios à l'époque des mariages ou de la fête de Diwali. La jeune génération, nous dit-on, est en demande d'effets spéciaux, de montage, de collage et de surimposition. Toutes ces images ont en commun, selon l'auteur, d'inverser le privilège accordé, depuis Pierce, par la sémiotique à la photo sur la peinture, dans la mesure où la première fait primer le montage et l'ornement sur la réalité. Outre les photos de mariage, on trouve des images où les individus s'intègrent dans celles de divinités, incorporant ainsi un peu de leur pouvoir. On peut aussi s'inclure dans un paysage Goa, le Cachemire, la Suisse-, autant de cadres qui servent de support à une «exploration personnelle d'une infinité d'alter egos». Parmi les exemples les plus courants, notons, outre les poses filmi (empruntées aux acteurs des films hindis, comme la college girl), la popularité des costumes régionaux et l'adoption, dans maintes photos, des signes de la modernité : une paysanne orissa arbore une montre à quartz et des lunettes de soleil; un villageois en tunique (dhoti) traverse sur sa moto (bullet) un paysage urbain qui pourrait être aussi bien San Francisco que Bombay. Dans un autre registre, un individu apparaît en martyr de l'indépendance (freedom fighter). Ces mises en scènes de soi, où il est question de "changement, de métamorphose, de tension et contradiction", viennent soutenir la négociation/reconfiguration des identités individuelles.

8 Par comparaison avec la photo française étudiée par Bourdieu dans les années 60 , les habitants de Nagda sont moins soucieux de « célébrer des positions sociales » que de les parodier ou de les transcender. Comme l'avait déjà noté Mac Dougall, ce type de photographie «augmente » (increase) son sujet. Comparable au cinéma auquel elle se réfère continuellement, elle est plus préoccupée de créer de nouveaux mondes que de dupliquer un monde existant. Il en résulte une conception particulière de la personne, où les "corps et les visages sont infiniment multiples et contingents", Pinney 
empruntant à MacKim Mariott l'idée d'un «dividuel » ${ }^{7}$, c'est-à-dire d'une identité "perméable, composite, partiellement divisible et transmissible» (p. 195). On peut toutefois se demander si, dans les « déplacements " photographiques des habitants de Nagda, le lien entre le visible et l'invisible, l'intérieur et l'extérieur n'est pas encore plus relâché que la notion de "dividuel » ne le laisse supposer. Contrairement à la tradition du portrait qui avait influencé la photographie officielle et la Galerie des grands hommes, on ne pense pas ici que la photo puisse capturer autre chose que le style, l'intériorité (charitra) d'une personne restant invisible. C'est à cette condition, d'après Pinney, qu'une telle liberté dans les montages a pu s'exercer. À l'opposé de Barthes à la recherche, dans La Chambre Claire, d'une photo de sa mère qui en incarnerait l'essence profonde, la pratique photographique étudiée ici ne repose pas sur le présupposé que le corps est la manifestation de l'intériorité, mais plutôt qu'il est un signe fluide capable de soutenir des « intériorités virtuelles » (imaginary interiorities). D'où l'étonnement que procure la dernière rencontre de l'auteur avec un photographe spécialisé dans les corrections de photos post mortem : apportez à Nanda Kishore une photo de votre ancêtre dans laquelle vous voulez lui voir porter un autre tilak (marque rituelle), un nouveau topi (chapeau) ou pagri (turban), ou dont vous estimez la coiffe trop haute, trop basse, le col de chemise trop ouvert, le rasage trop approximatif, et il vous fera la correction désirée! N'y a-t-il pas là un beau pied-de-nez à l'obsession d'authenticité de la photographie coloniale?

Il s'agit là d'un ouvrage fort, tant en ce qui concerne la modernité indienne, dont on saisit la crétivité, que le statut épistémologique accordé à l'image ${ }^{8}$. Christopher Pinney réussit par ailleurs un pari méthodologique important qui n'est pas sans rappeler le travail d'Emma Tarlo sur le vêtement ${ }^{9}$ : comme elle, il se fait historien, multiplie ses points d'ancrage empiriques et parvient à brosser une complexité sociohistorique qui prend la forme d'une imbrication de jeux d'images autour des personnes et des institutions. L'approche des objets culturels, et particulièrement du lien entre image et société, en ressort rafraîchie.

\section{NOTES}

1. David Mac Dougall, « Photo Hierarchicus : Signs and Mirrors in Indian Photography », Visual Anthropology, 1992, V ; cf. aussi David \& Judith Mac Dougall, Photowallahs, film documentaire, 1992.

2. Pierre Bourdieu, Un art moyen, Paris, Minuit, 1965.

3. Arjun Appadurai, The Social Life of Things. Commodities in Cultural Perspective, Cambridge, Cambridge University Press, 1986.

4. L'expression est de Nicholas Thomas dans son livre, Entangled Object. Exchange, Material Culture and Colonialism in the Pacific, Cambridge, Mass., Harvard University Press, 1991.

5. Le terme darshan, "vision », désigne le fait de voir et d'être vu par la divinité.

6. Judith Mara Gutman, Through Indian Eyes. 19th and Early 20th Century Photography from India, New York, 1982. 
7. MacKim Mariott, «Interpreting Indian Society. A Monistic Alternative to Dumont's Dualism », Journal of Asian Studies, 1976, 36 (3).

8. Sur la place de l'image dans le raisonnement anthropologique, voir Marcus Banks \& Howard Morphy, eds, Rethinking Visual Anthropology, London, Yale University Press, 1999, et Bruno Latour, Paris : ville invisible, Paris, Synthélabo, 1998.

9. Emma Tarlo, Clothing Matters. Dress and Identity in India, Delhi, Vikas, 1996.

\section{AUTEUR}

\section{EMMANUEL GRIMAUD}

CNRS, Maison de l'archéologie et de l'ethnologie, Université Paris-X, Nanterre. 